\title{
The role of "penumbra sign" and diffusion-weighted imaging in adnexal masses: do they provide a clue in differentiating tubo-ovarian abscess from ovarian malignancy?
}

\author{
Ranjan Kumar Patel ${ }^{1, B, C, E, F}$, Anju Garg ${ }^{1, A, D}$, Rashmi Dixit ${ }^{1, A, C, D, E}$, Gauri Gandhi' ${ }^{2, D}$, Nita Khurana ${ }^{3, B, D, E}$ \\ 'Department of Radiodiagnosis, Maulana Azad Medical College, New Delhi, India \\ 2Department of Obstetrics and Gynaecology, Maulana Azad Medical College, New Delhi, India \\ ${ }^{3}$ Department of Pathology, Maulana Azad Medical College, New Delhi, India
}

\section{Abstract}

Purpose: To evaluate the role of "penumbra sign", diffusion-weighted imaging (DWI), and the apparent diffusion coefficient (ADC) value in differentiating tubo-ovarian abscess (TOA) from ovarian malignancy.

Material and methods: Thirty-six patients with 50 adnexal masses (tubo-ovarian abscess, $n=24$; ovarian malignancy, $n=26$ ), who underwent magnetic resonance imaging (MRI) with DWI, were retrospectively evaluated. "Penumbra sign" (hyperintense rim on T1W images), diffusion restriction, and mean apparent diffusion coefficient (ADC) values from cystic (c-ADC) and solid (s-ADC) components were evaluated for all the masses.

Results: "Penumbra sign" on T1W images was significantly more common in the TOA group $(n=21,87.5 \%)$ than in the ovarian malignancy group $(n=2,7.7 \%)(p<0.001)$. Similarly, diffusion restriction in the cystic component was more frequent in the TOA group $(n=24,100 \%$ vs. $n=2,10.5 \%$; $p<0.001)$. In contrast, diffusion restriction in the solid component was more common in the ovarian malignancy group ( $n=5,20.8 \%$ vs. $n=26,100 \%$; $p<0.001)$. The mean c-ADC value was significantly lower in TOAs $(p<0.001)$. A c-ADC value of $1.31 \times 10^{-3} \mathrm{~mm}^{2} / \mathrm{s}$ may be an optimal cut-off in distinguishing TOAs from ovarian malignancies. Conversely, the mean s-ADC value was significantly lower in the ovarian malignancy group $(p<0.001)$. An s-ADC value of $0.869 \times 10^{-3} \mathrm{~mm}^{2} / \mathrm{s}$ may be an optimal cut-off in differentiating ovarian malignancies from TOAs $(p<0.001)$. ROC curve analysis showed that c-ADC values had a higher diagnostic accuracy than s-ADC values.

Conclusions: "Penumbra sign" on T1W images, diffusion characteristics, and ADC values provide important clues in addition to conventional MR imaging features in differentiating TOA from ovarian malignancy.

Key words: penumbra sign, diffusion-weighted imaging (DWI), conventional MRI, apparent diffusion coefficient (ADC), tubo-ovarian abscess (TOA), ovarian malignancy.

\section{Introduction}

A tubo-ovarian abscess (TOA) is a complication of pelvic inflammatory disease (PID). Typical clinical presentations of TOAs include fever, chills, pelvic pain, and adnexal ten- derness. Laboratory parameters show leucocytosis, elevated erythrocyte sedimentation rate (ESR), and C-reactive protein (CRP). However, about $20 \%$ of the patients do not show any typical signs and symptoms [1]. Other signs and symptoms like abdominal pain, vaginal discharge, and abnormal vaginal bleeding are non-specific and can be seen

Correspondence address:

Ranjan Kumar Patel, Assistant Professor, Department of Radiodiagnosis, AlIMS, Bhubaneswar, PIN-751019, Odisha, India, e-mail: ranjanair1@gmail.com

Authors' contribution:

A Study design · B Data collection · C Statistical analysis · D Data interpretation · E Manuscript preparation · F Literature search · G Funds collection 
in patients with other adnexal lesions [2]. CA-125 levels are also non-specific and can be elevated in patients with TOA and ovarian malignancy [3].

A TOA involves the fallopian tube, ovary, and adjacent pelvic soft tissue. It often appears as a complex adnexal mass with a multilocular or mixed solid-cystic appearance on imaging [4]. The co-existence of several imaging features, such as thickened wall and septae, ill-defined margins, solid enhancing areas, lymphadenopathy, ascites, and peritoneal and omental thickening, can make it challenging to differentiate a TOA from an ovarian malignancy, particularly in developing countries where pelvic inflammatory disease and tuberculosis are common [5]. However, distinguishing one from the other is very important for the initiation of appropriate treatment. TOAs need early antibiotic therapy with or without abscess drainage, whereas ovarian malignancies need a multidisciplinary action, such as chemotherapy, surgery, or both [6].

Due to ongoing inflammation, TOA forms a layer of granulation tissue along the inner walls of the abscess cavity like that of abscesses in other places, such as liver abscess, soft tissue abscess, and subacute osteomyelitis $[4,7,8]$. This granulation tissue appears as a hyperintense rim on T1W images due to the presence of microhaemorrhages $[4,8]$. A similar hyperintense rim on T1W images in Brodie's abscess has typically been described as the "penumbra sign" [8]. In addition to other imaging features on conventional MR images, the "penumbra sign" could be an important feature that differentiates a TOA from an ovarian malignancy.

Although MRI is the imaging modality of choice to characterize adnexal masses, conventional MR sequences may not always be able to differentiate a TOA and an ovarian malignancy due to an overlap in morphological features [9]. Positron emission tomography with computed tomography (PET-CT) also has a low specificity owing to FDG uptake in both TOA and ovarian malignancy [10]. In such cases, additional imaging sequences such as diffusion and dynamic contrast-enhanced MRI could provide functional information that helps in better lesion characterization [11].

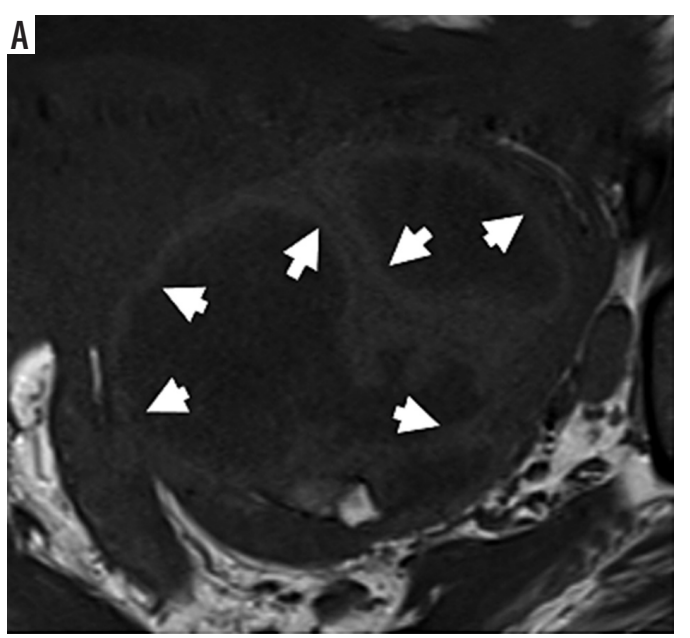

Cavities in TOAs contain purulent materials and debris, unlike the cystic components of malignant ovarian lesions. It is a known fact that pus shows diffusion restriction with low apparent diffusion coefficient (ADC) values [12]. This property has been widely used in diagnosing and differentiating brain abscesses from necrotic brain tumours [12]. Malignancies do not show granulation tissue, unlike abscesses. However, solid areas of malignant tumours often exhibit marked diffusion restriction due to high cellularity [11].

Several studies have evaluated the role of diffusionweighted imaging (DWI) and ADC values in differentiating benign from malignant adnexal masses, with conflicting results $[13,14]$. There are limited studies with a head-tohead comparison of diffusion characteristics between TOAs and ovarian malignancies $[10,15,16]$.

This study aims to evaluate whether the "penumbra sign" on T1W images, diffusion restriction, and ADC values can provide important clues in distinguishing a TOA from an ovarian malignancy.

\section{Material and methods}

We conducted a retrospective study after approval from the institutional review board. A total of 58 patients diagnosed either with ovarian malignancy or TOA from June 2019 to July 2020 were identified from the hospital database. The final diagnosis was confirmed by pathological examination or culture of aspiration material. Thirty-six of these patients who underwent contrast-enhanced MRI with DWI ( $b$ values: 50,400 , and $800 \mathrm{~s} / \mathrm{mm}^{2}$ ) on a 3-Tesla MR system (MAGNETOM Skyra) in our department were included in the study. Twenty-two patients were excluded for the following reasons: unavailability of complete clinical/laboratory data, poor image quality, or prior treatment history for TOA or ovarian malignancy. Finally, a total of 50 adnexal lesions in 36 patients (bilateral lesions in 14 women) were included $(n=50)$. Patients' age, clinical symptoms and signs, and laboratory parameters, including tumour markers, were recorded.

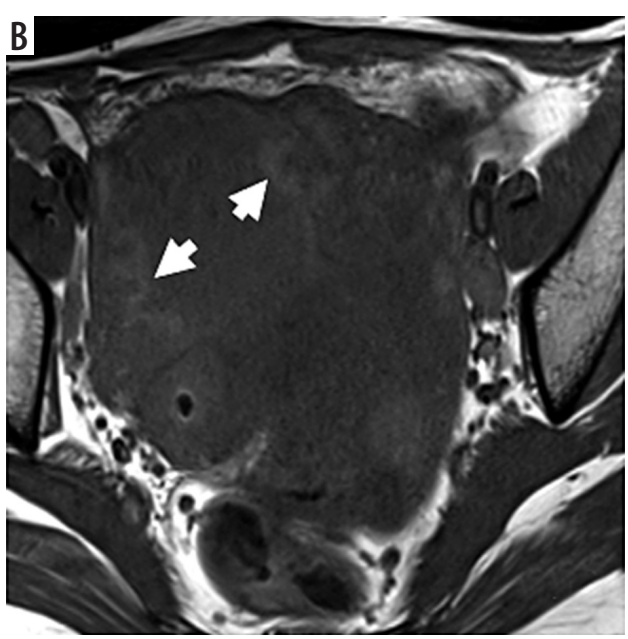

Figure 1. Penumbra sign on T1-weighted images. A) Right tubo-ovarian abscess shows a complex adnexal mass with hyperintense rim along the inner walls of cavities (white arrows) - termed as "penumbra sign". B) The cavity of tubo-ovarian abscess in another patient also shows hyperintense rim (white arrows) 
Conventional MR images were evaluated for morphological features, signal characteristics, and enhancement pattern of the adnexal mass, peritoneal thickening, omental caking, distance metastases, ascites, and lymphadenopathy. Thickened walls and septae $(>3 \mathrm{~mm})$, vegetations, and varying degrees of solid portions, showing post-contrast enhancement, were identified as solid components [17]. A hyperintense lining along the inner aspect of the cystic component on T1W images was termed as the "penumbra sign" (Figure 1).

Diffusion restriction was defined as high signal intensity on DWI at $b=800 \mathrm{~s} / \mathrm{mm}^{2}$ and low signal on the corresponding ADC map. Solid and cystic components were analysed separately for diffusion restriction. Similarly, quantitative ADC values of solid and cystic areas were calculated separately by placing regions of interest (ROIs) on the ADC map. Several ROIs were drawn in each lesion, and the lowest ADC values of cystic (c-ADC) and solid (s-ADC) components were recorded.

Two radiologists (a genitourinary subspecialist with more than 10 years of experience and a final-year resident) independently evaluated the presence of "penumbra sign," diffusion characteristics, and ADC values.

\section{Statistical analysis}

The kappa ( $\mathrm{\kappa}$ )-value and intraclass correlation coefficient (ICC) were used to assess the interobserver agreement for qualitative variables and $\mathrm{ADC}$ of lesions, respectively. Differences in opinion were settled by consensus. The reproducibility between observers was considered to be good when the $\kappa$-value and ICC were greater than 0.8. Statistical significance for the intergroup (TOA and ovarian malignancy) was evaluated by $\chi^{2}$ test or Fisher's exact test depending on the expected frequencies. For the quantitative analysis, Student's $t$-test or Mann-Whitney $U$ test was used depending on the distribution of data. Receiver operating characteristics (ROC) curve analysis was performed to determine the diagnostic accuracy and optimal cut-off of the apparent diffusion coefficient (ADC). A $p$-value of $<0.05$ was considered statistically significant. All analyses were carried out using IBM SPSS statistics (v. 23.0. Armonk, NY).

\section{Results}

Thirty-six patients with 50 adnexal masses were analysed, including 24 TOA in 18 patients and 26 ovarian malignancies in 18 patients. Table 1 enlists the final diagnosis of lesions. Out of 24 TOAs, 9 (37.5\%) were pyogenic and $15(62.5 \%)$ were tubercular. Serous cystadenocarcinoma $(n=10,61.5 \%)$ was the most common type among the ovarian malignancies.

The demographic and clinical profile of the study cohort is shown in Table 2. Patients of TOAs were younger than those with ovarian malignancy $(28.5 \pm 7.9$ vs. 45.8 \pm 14.2 years; $p<0.001$ ). There was an overlap in clinical signs and symptoms between the 2 groups. Adnexal tenderness and leucocytosis were more common in patients with TOAs $(p<0.05)$. Serum CA-125 level was elevated in both groups. However, a level of $>100 \mathrm{IU} / \mathrm{ml}$ was more frequently seen in ovarian malignancy patients $(p=0.016)$ and was seen in cases of serous cystadenocarcinoma. The carcinoembryonic antigen (CEA) level was elevated in 2 patients of mucinous cystadenocarcinoma, and the betahuman chorionic gonadotrophin ( $\beta$-hCG) level was raised in a patient with dysgerminoma.

\section{Morphological magnetic resoannce features}

Conventional MR imaging features are summarised in Table 3. The size of lesions in the ovarian malignancy group $(9.75 \pm 3.98 \mathrm{~cm})$ was larger than that of the TOA group $(5.45 \pm 1.54 \mathrm{~cm})(p<0.001)$. All the lesions in our cohort were solid-cystic except 7 malignant ovarian masses, which were entirely solid. Multilocularity $(87.5 \%$ vs. $61.5 \%)$ and the presence of a dilated fallopian tube (70.8\% vs. $19.2 \%)$ were significantly more frequent in TOAs as compared to ovarian malignancy.

There was excellent interobserver agreement for the "penumbra sign" ( $\kappa=0.813)$, as shown in Table 4. "Penumbra sign" was also significantly more common in the TOA group ( $n=21,87.5 \%$ ) (Figures 2 and 3 ). In contrast, only 2 malignant lesions demonstrated a hyperintense rim on T1WI $(p<0.001)$. "Penumbra sign" had a sensitivity, specificity, positive predictive value (PPV), and negative predictive value (NPV) of $87.5 \%, 89.5 \%, 91.3 \%$, and $85 \%$,

Table 1. Diagnosis based on pathological findings

\begin{tabular}{|l|c|c|c|c|}
\hline \multirow{2}{*}{ Category } & Diagnosis & $\begin{array}{c}\text { Number } \\
\text { of patients }\end{array}$ & $\begin{array}{c}\text { Number } \\
\text { of lesions }\end{array}$ & \% (within each group) \\
\hline \multirow{3}{*}{ Tubo-ovarian abscess $(n=24)$} & Pyogenic & 7 & 9 & 37.5 \\
\cline { 2 - 5 } & Tubercular & 11 & 15 & 62.5 \\
\hline \multirow{3}{*}{ Ovarian malignancy $(n=26)$} & Serous cystadenocarcinoma & 10 & 16 & 61.5 \\
\cline { 2 - 5 } & Mucinous cystadenocarcinoma & 3 & 4 & 15.3 \\
\cline { 2 - 5 } & Granulosa cell tumour & 3 & 3 & 11.5 \\
\cline { 2 - 5 } & Dysgerminoma & 1 & 1 & 3.8 \\
\cline { 2 - 5 } & Krukenberg tumour & 1 & 2 & 7.7 \\
\hline
\end{tabular}


Table 2. Demographic and clinical profile of patients

\begin{tabular}{|c|c|c|c|}
\hline Parameters & Tubo-ovarian abscess $(n=18)$ & Ovarian malignancy $(n=18)$ & $p$-value \\
\hline Age in years $($ mean $\pm S D)$ & $28.5 \pm 7.9$ & $45.8 \pm 14.2$ & $<0.001$ \\
\hline \multicolumn{4}{|l|}{ Symptoms and signs, $n(\%)$} \\
\hline Fever & $5(27.8)$ & $1(5.6)$ & 0.07 \\
\hline Lower abdominal pain & $13(72.2)$ & $14(77.8)$ & 0.70 \\
\hline Abnormal vaginal discharge & $9(50.0)$ & $4(22.2)$ & 0.08 \\
\hline Abnormal uterine bleeding & $3(16.7)$ & $7(38.9)$ & 0.13 \\
\hline Adnexal tenderness & $15(83.3)$ & $5(27.8)$ & $<0.001$ \\
\hline \multicolumn{4}{|l|}{ Laboratory parameters, $n$ (\%) } \\
\hline Elevated WBC counts & $4(22.2)$ & 0 & 0.03 \\
\hline Elevated C-reactive protein (> $10 \mathrm{mg} / \mathrm{l}$ ) & $7(38.9)$ & $2(11.1)$ & 0.054 \\
\hline \multicolumn{4}{|l|}{ Tumour markers, $n(\%)$} \\
\hline \multicolumn{4}{|l|}{ Elevated CA-125 (> $35 \mathrm{IU} / \mathrm{ml})$} \\
\hline$<100 \mathrm{IU} / \mathrm{ml}$ & $6(33.3)$ & $1(5.6)$ & \multirow[t]{2}{*}{0.016} \\
\hline$>100 \mathrm{IU} / \mathrm{ml}$ & $3(16.6)$ & $9(50.0)$ & \\
\hline Elevated CEA (> $5 \mathrm{ng} / \mathrm{ml})$ & 0 & $2(11.1)$ & - \\
\hline Elevated $\beta$-hCG (> $5 \mathrm{mlU} / \mathrm{ml})$ & 0 & $1(2.7)$ & - \\
\hline
\end{tabular}

respectively, in differentiating TOA from ovarian malignancy.

The mean wall/septal thickness, fluid-fluid levels, and signal intensity of solid and cystic components did not show any significant difference between the 2 groups.

Peritoneal thickening and enhancement, omental caking, lymphadenopathy, ascites, and involvement of other organs were seen in both TOA and ovarian malignancy. Among these features, only free ascites was statistically more common in ovarian malignancy. Loculated ascites was seen only in $27.8 \%$ of TOA patients.

\section{Diffusion characteristics}

There was excellent interobserver agreement for diffusion restriction of cystic $(\kappa=1)$ and solid $(\kappa=0.957)$ components. Similarly, excellent interobserver agreement was also noted for the ADC measurements of the cystic (ICC $=0.984 ; 95 \% \mathrm{CI}: 0.970-0.991)$ and solid $(\mathrm{ICC}=0.943$; 95\% CI: 0.760-0.978) components (Table 4).

\section{Diffusion characteristics in cystic component}

Diffusion characteristics and mean ADC values are summarized in Table 5. A cystic component was seen in 19 out of 26 malignant masses, and only 2 (10.5\%) of them showed diffusion restriction in their cystic component $(p<0.001)$ (Figures 4 and 5). Conversely, all the TOA group masses showed diffusion restriction in cystic components (Figures 2 and 3). The diffusion restriction in cystic components had a specificity of $89.5 \%$ and PPV of $92.3 \%$ with $100 \%$ sensitivity and NPV for diagnosing
TOAs. Diffusion restriction in the cystic components of the 2 malignant masses (serous cystadenocarcinoma $[n=1]$ and granulosa cell tumour $[n=1]$ ) was due to haemorrhage, which was identified by marked hyperintensity on fat-saturated T1W images. The difference in mean c-ADC values between TOA and ovarian malignancy group was statistically significant $(p<0.001)$. The mean c-ADC value in the TOA group was 0.779 $\pm 0.317 \times 10^{-3} \mathrm{~mm}^{2} / \mathrm{s}$, and that of ovarian malignancy was $2.368 \pm 0.698 \times 10^{-3} \mathrm{~mm}^{2} / \mathrm{s}$. The ROC curve analysis showed an optimal cut-off ADC value of $1.31 \times 10^{-3} \mathrm{~mm}^{2} / \mathrm{s}$ (AUC $=0.932 ; 95 \%$ CI: $0.837-1.00)$ with a sensitivity of $95.8 \%$ and specificity of $89.5 \%$ in differentiating TOA from ovarian malignancy (Figure 6A).

\section{Diffusion characteristics in solid component}

In all the cases of ovarian malignancy, solid areas showed diffusion restriction as opposed to only 5 (20.8\%) TOAs $(p<0.001)$ (Figures 4 and 5). Out of those 5 TOAs, 2 were pyogenic, and 3 were tubercular abscesses. Diffusion restriction in solid components had a specificity of 79.2\% and PPV of $83.9 \%$ with $100 \%$ sensitivity and NPV in diagnosing ovarian malignancies. The mean s-ADC value in the ovarian malignancy group was $0.760 \pm 0.129$ $\times 10^{-3} \mathrm{~mm}^{2} / \mathrm{s}$, and that in the TOA group was 1.041 $\pm 0.192 \times 10^{-3} \mathrm{~mm}^{2} / \mathrm{s}$, which showed a statistical significance $(p<0.001)$ (Table 4$)$. The ROC curve analysis showed that a cut-off ADC value of $0.869 \times 10^{-3} \mathrm{~mm}^{2} / \mathrm{s}$ (AUC $=0.908 ; 95 \%$ CI: $0.830-0.985)$ had a sensitivity of $80.8 \%$ and specificity of $79.2 \%$ in differentiating ovarian malignancy from TOA (Figure 6B). 
Table 3. Imaging features on conventional magnetic resonance sequences

\begin{tabular}{|c|c|c|c|}
\hline \multirow[t]{2}{*}{ Variables } & \multicolumn{2}{|c|}{ Morphological features of lesion } & \multirow[t]{2}{*}{$p$-value } \\
\hline & Tubo-ovarian abscess, $n=24$ & Ovarian malignancy, $n=26$ & \\
\hline Longest dimension (cm) & $5.45 \pm 1.54$ & $9.75 \pm 3.98$ & $<0.001$ \\
\hline \multicolumn{4}{|l|}{ Appearance, $n(\%)$} \\
\hline Solid-cystic & $24(100)$ & $19(73.1)$ & \multirow[t]{2}{*}{0.004} \\
\hline Purely solid & 0 & $7(26.9)$ & \\
\hline Multilocularity, $n(\%)$ & $21(87.5)$ & $16(61.5)$ & 0.038 \\
\hline Dilated fallopian tubes, $n$ (\%) & $17(70.8)$ & $5(19.2)$ & $<0.001$ \\
\hline Fluid-fluid level, $n(\%)$ & $6(25.0)$ & $5(19.2)$ & 0.62 \\
\hline Papillary projections, $n(\%)$ & $5(20.8)$ & $8(30.8)$ & 0.42 \\
\hline Mean wall/septae thickness (mm) & $2.8 \pm 0.72$ & $2.9 \pm 1.21$ & 0.7 \\
\hline \multicolumn{4}{|l|}{ T2 signal of solid tissue, $n(\%)$} \\
\hline Hyperintense & $16(66.7)$ & $24(92.3)$ & \multirow[t]{2}{*}{0.2} \\
\hline Iso-hypointense & $4(16.7)$ & $2(7.7)$ & \\
\hline T1 hyperintense rim ("penumbra sign"), $n(\%)$ & $21(87.5)$ & $2(7.7)$ & $<0.001$ \\
\hline \multicolumn{4}{|l|}{ T1 signal of cystic part, $n(\%)$} \\
\hline Hyperintense & $4(16.7)$ & $4(15.4)$ & \multirow[t]{2}{*}{0.7} \\
\hline Iso-hypointense & $20(83.3)$ & $15(57.7)$ & \\
\hline \multicolumn{4}{|l|}{ T2 signal of cystic part, $n(\%)$} \\
\hline Hyperintense & $21(87.5)$ & $18(69.2)$ & \multirow[t]{2}{*}{0.8} \\
\hline Iso-hypointense & $3(12.5)$ & $2(7.7)$ & \\
\hline Associated features, $n(\%)$ & $n=18$ & $n=18$ & $p$-value \\
\hline Peritoneal thickening and enhancement & $14(77.8)$ & $12(66.7)$ & 0.45 \\
\hline Omental caking & $5(27.8)$ & $10(55.6)$ & 0.09 \\
\hline Lymphadenopathy & $11(61.1)$ & $12(66.7)$ & 0.72 \\
\hline Free ascites & $3(16.7)$ & $12(66.7)$ & 0.002 \\
\hline Loculated ascites & $5(27.8)$ & 0 & 0.016 \\
\hline Other organs involvement & $2(11.1)$ & $3(16.7)$ & 0.63 \\
\hline
\end{tabular}

Table 4. Interobserver agreements for "penumbra sign", diffusion restriction, and ADC values

\begin{tabular}{|c|c|c|c|c|}
\hline Variables & Observer 1 & Observer 2 & $\kappa$-value & $p$-value \\
\hline Penumbra sign & $\mathrm{TOA}=21$ & $\mathrm{TOA}=22$ & 0.813 & $<0.001$ \\
\hline (Hyperintense rim on T1WI) & $0 M=2$ & $0 M=1$ & & \\
\hline \multicolumn{5}{|c|}{ Diffusion restriction $\left(b=800 \mathrm{~s} / \mathrm{mm}^{2}\right)$} \\
\hline \multirow[t]{2}{*}{ Solid component (sDWI) } & $\mathrm{TOA}=5$ & $\mathrm{TOA}=6$ & \multirow[t]{2}{*}{0.957} & \multirow[t]{2}{*}{$<0.001$} \\
\hline & $0 M=26$ & $0 M=26$ & & \\
\hline \multirow[t]{2}{*}{ Cystic component (CDWI) } & $\mathrm{TOA}=24$ & $\mathrm{TOA}=24$ & \multirow[t]{2}{*}{1.00} & \multirow[t]{2}{*}{$<0.001$} \\
\hline & $0 M=2$ & $0 M=2$ & & \\
\hline Variables & Observer 1 & Observer 2 & Interclass correlation coefficient ( $95 \% \mathrm{Cl})$ & $p$-value \\
\hline \multicolumn{5}{|c|}{ Mean $A D C$ value (mean $\pm S D \times 10^{-3} \mathrm{~s}$ ) } \\
\hline Solid component (sADC) & $0.895 \pm 0.214$ & $0.965 \pm 0.253$ & $0.943(0.760-0.978)$ & $<0.001$ \\
\hline Cystic component (CADC) & $1.481 \pm 0.949$ & $1.542 \pm 0.923$ & $0.984(0.970-0.991)$ & $<0.001$ \\
\hline
\end{tabular}

TOA - tubo-ovarian abscess, OM - ovarian malignancy, ICC - interclass correlation coefficient 

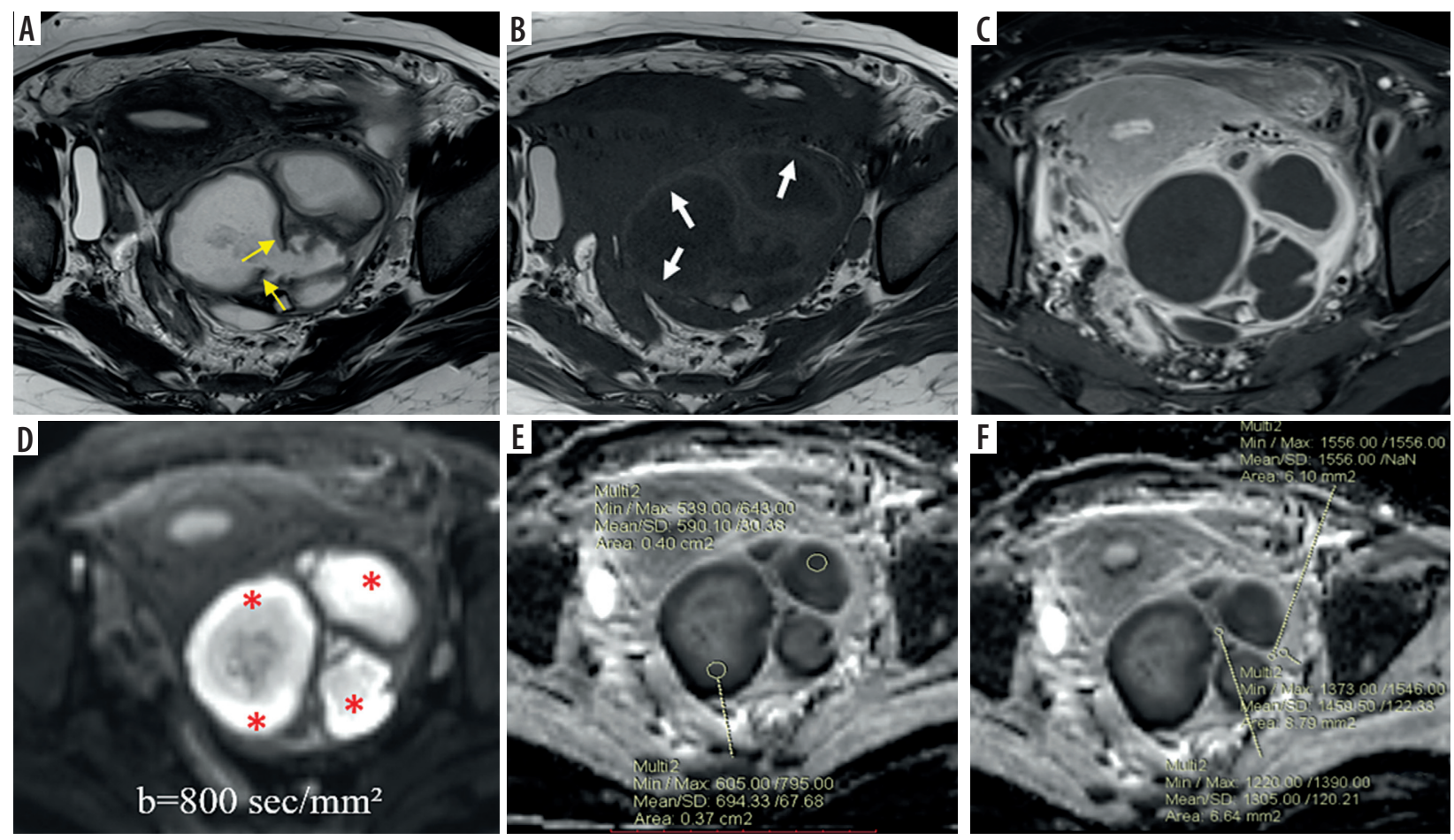

Figure 2. Left-sided tubo-ovarian abscess in a 21-year-old female. A) Axial T2W image shows a complex solid-cystic right adnexal mass with a few incomplete septae (yellow arrows). B) Axial T1W image shows "penumbra sign" (white arrows B). C) CE-T1W image depicts enhancing thick irregular septae, solid peripheral component, and irregular enhancement in the surrounding. D) DWl image showing marked diffusion restriction in the abscess cavity (red asterisks) with (E) low $A D C$ values (minimum $A D C$ of $0.560 \times 10^{-3} \mathrm{~mm}^{2} / \mathrm{s}$ ); $\mathrm{F}$ ) solid component appears hypointense on DWI with higher $A D C$ values (minimum value of $1.305 \times 10^{-3} \mathrm{~mm}^{2} / \mathrm{s}$ )
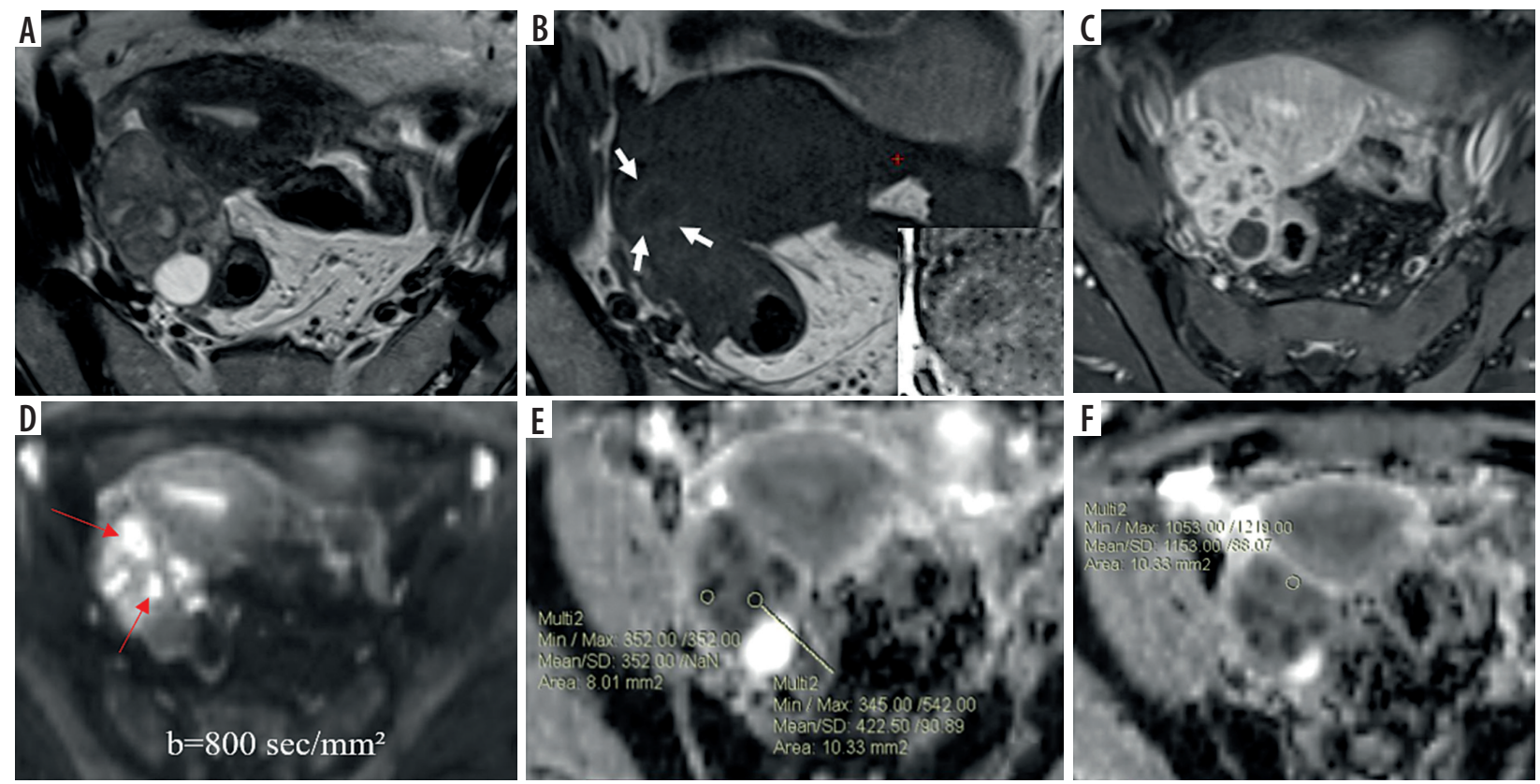

Figure 3. Right tubo-ovarian abscess (tubercular) in a 32-year-old female. A) Axial T2W image shows a complex solid cystic right adnexal mass of intermediate signal intensity. B) Axial T1W image at a slightly lower level shows "penumbra sign" around the abscess cavity (white arrows). C) Post-contrast T1WI image showing enhancing solid components. D) DWI image shows marked diffusion restriction in cystic areas (red arrows) with a minimum $A D C$ value of $0.352 \times 10^{-3} \mathrm{~mm}^{2} / \mathrm{s}(\mathrm{E})$, whereas solid tissue $(\mathbf{F})$ does not show diffusion restriction with an $A D C$ value of $1.153 \times 10^{-3} \mathrm{~mm}^{2} / \mathrm{s}$

The sensitivity and specificity of different variables are summarized in Table 6. Using ROC curve analysis, the $\mathrm{ADC}$ values in cystic components (c-ADC) had a higher diagnostic accuracy than the ADC values in solid components (s-ADC) in differentiating TOAs from ovarian malignancy.

\section{Discussion}

Although the presence of typical signs and symptoms with a complex adnexal mass on imaging favours TOA, only a few patients with TOA show characteristic clinical features, such as fever, adnexal tenderness, leucocytosis, 
Table 5. Comparison of diffusion characteristics and ADC values

\begin{tabular}{|c|c|c|c|}
\hline Variables & Tubo-ovarian abscess & Ovarian malignancy & $p$-value \\
\hline \multicolumn{4}{|c|}{ Diffusion restriction $\left(b=800 \mathrm{~s} / \mathrm{mm}^{2}\right)$} \\
\hline Cystic component & $24 / 24(100 \%)$ & $2 / 19(10.5 \%)$ & $<0.001$ \\
\hline Solid component & $5 / 24(20.8 \%)$ & $26 / 26(100 \%)$ & $<0.001$ \\
\hline \multicolumn{4}{|c|}{ Mean $A D C$ value (mean $\pm S D \times 10^{-3} \mathrm{~mm}^{2} / \mathrm{s}$ ) } \\
\hline Cystic component (CADC) & $0.779 \pm 0.317$ & $2.368 \pm 0.698$ & $<0.001$ \\
\hline Solid component (SADC) & $1.041 \pm 0.192$ & $0.760 \pm 0.129$ & $<0.001$ \\
\hline
\end{tabular}
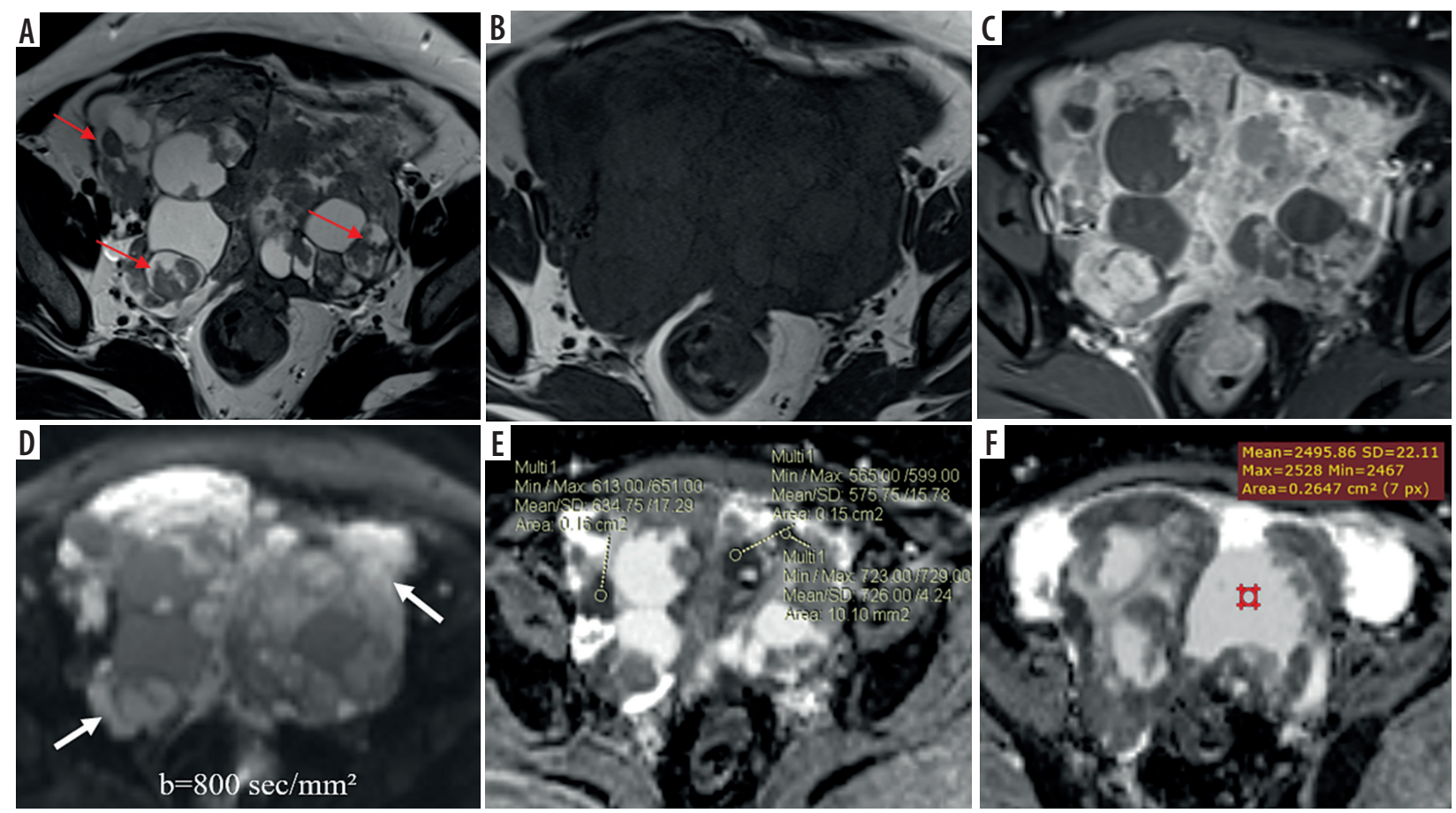

Figure 4. Bilateral ovarian serous cystadenocarcinoma. Axial T2W (A) and T1W (B) images show complex T2 intermediate and T1 hypointense multilocular solid-cystic masses in bilateral adnexae with multiple papillary projections (red arrows A); no "penumbra sign" is noted; contrast-enhanced T1W image (C) shows enhancing solid components and septae. Axial DW image (D) reveals marked diffusion restriction in the solid areas (white arrows) with (E) low $A D C$ values (minimum $A D C$ value of $0.565 \times 10^{-3} \mathrm{~mm}^{2} / \mathrm{s}$ ); F) cystic component showing facilitated diffusion with higher ADC values (minimum value of $\left.2.495 \times 10^{-3} \mathrm{~mm}^{2} / \mathrm{s}\right)$

elevated ESR, and CRP $[1,18]$. In a real-life situation, most patients with TOA and ovarian malignancy present with non-specific symptoms and signs. Thus, it may not always be possible to differentiate TOA from ovarian malignancy based only on clinical and laboratory features $[1,2]$.

Features like dilated fallopian tubes and loculated ascites may be more indicative of a TOA. Our study also showed a higher incidence of dilated fallopian tubes on MR imaging among TOA than malignant ovarian lesions. In addition, loculated ascites was also seen only in patients with TOAs. Studies have shown that TOA and ovarian malignancy share many morphological features on conventional MR imaging, such as multilocularity, thickened septae and/or walls, and the presence of solid areas $[4,18]$. Additionally, TOA, especially tubercular, can mimic ovarian carcinomatosis due to associated peritoneal thickening, omental caking, and ascites. Lymphadenopathy and involvement of other organs are also not un- common in such patients [19]. As mentioned above, the overlap in these imaging features necessitates looking for other key imaging features on conventional MR sequences and the addition of functional imaging techniques, such as DWI and DCE-MRI, to resolve the diagnostic dilemma between TOAs and ovarian malignancies.

We found a "penumbra sign" in 21 of 24 (87.5\%) TOAs as opposed to only 2 of $26(10.5 \%)$ ovarian malignant lesions $(p<0.001)$. Penumbra sign alone had a sensitivity of $87.5 \%$ and specificity of $89.5 \%$ in distinguishing a TOA from an ovarian malignancy. The presence of microhaemorrhages with a layer of granulation tissue that forms along the inner wall of the abscess cavity explains the higher incidence of the "penumbra sign" in the TOA group. A study by Ha et al. reported the "penumbra sign" on T1W images in 8 out of 9 TOAs (88.9\%) [4]. Kim et al. also described the usefulness of the T1-hyperintense rim along the inner wall of the abscess cavity in characteriz- 

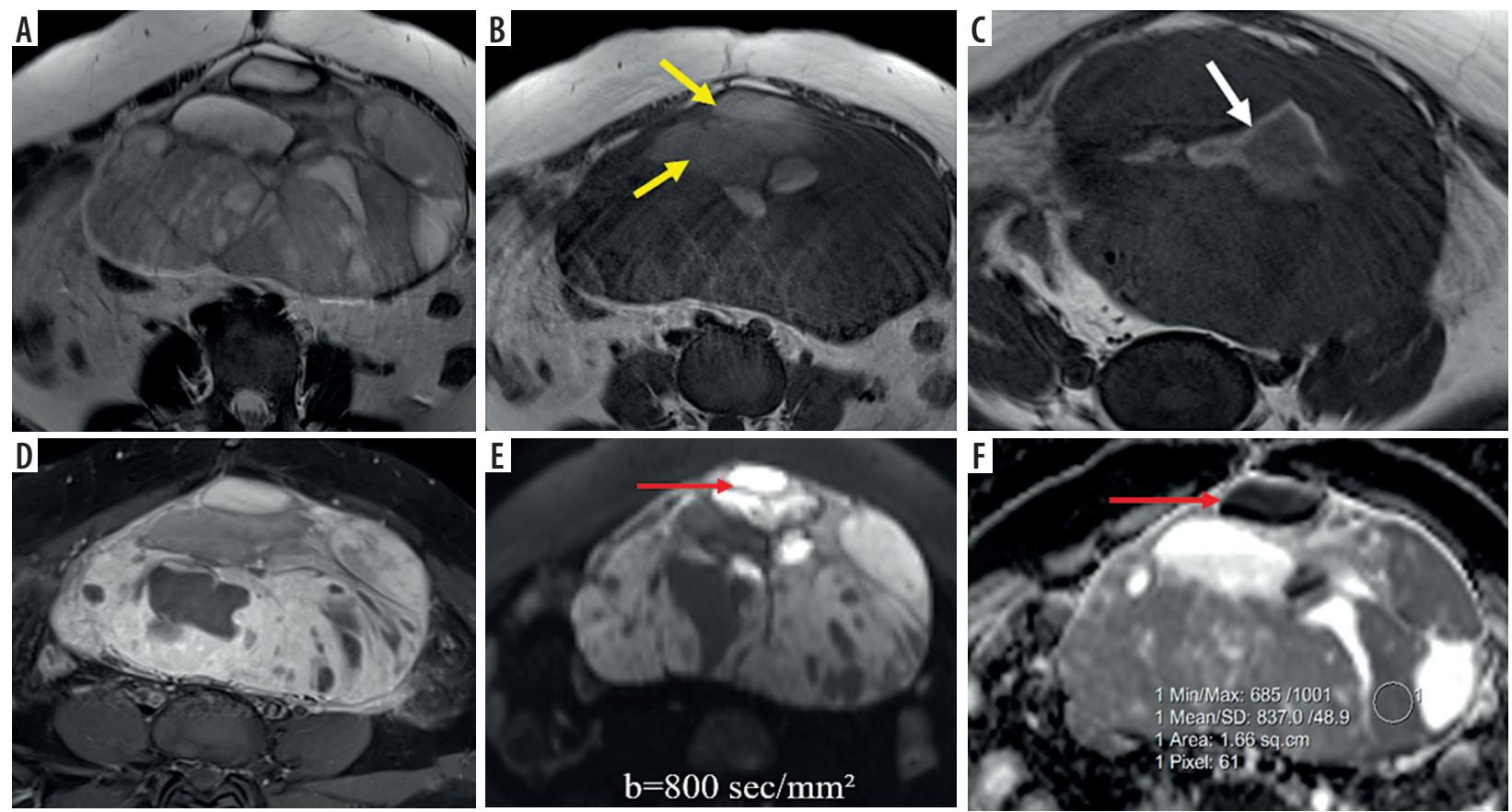

Figure 5. Juvenile granulosa cell tumour of the left ovary. Axial T2W (A) and T1W Non-FS (B) images show a well-defined T2 intermediate and T1 hypointense solid-cystic left ovarian mass; few T1 hyperintense cystic areas are noted (yellow arrows B), indicating haemorrhage; T1W image (C) at a lower section showing a T1 hyperintense rim around the cyst (white arrow C); post-contrast T1WI image (D) shows enhancing solid component; axial DWI image (E) depicts high signal in the solid as well as cystic (red arrows $E$ and $F$ ) areas with low signal on corresponding ADC map (F), suggesting diffusion restriction; $A D C$ value measured in the solid area is $0.837 \times 10^{-3} \mathrm{~mm}^{2} / \mathrm{s}$
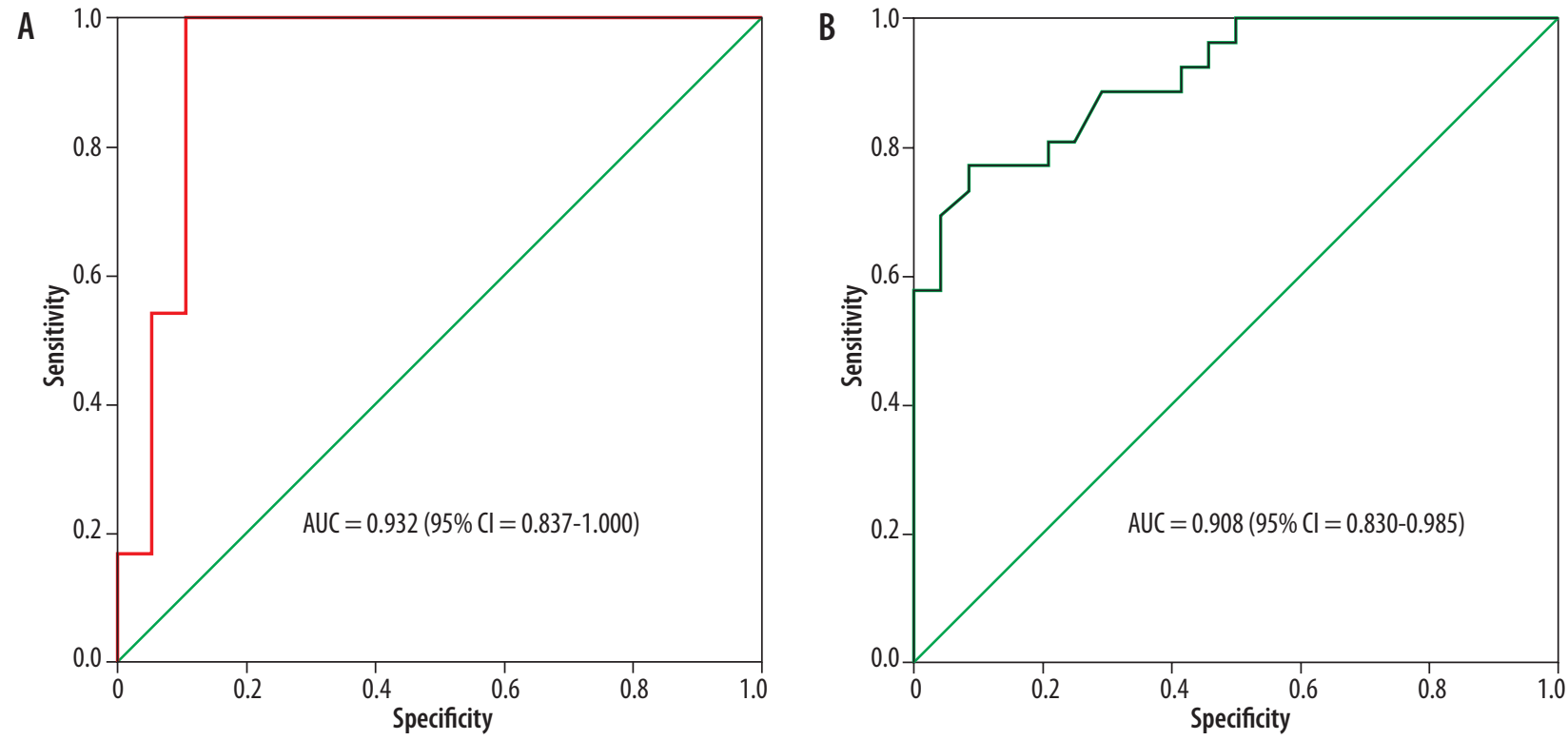

Figure 6. ROC curves analysis show greater area under the curve (AUC) for C-ADC values ( $A$ ) than that of s-ADC values (B), indicating that c-ADC has higher diagnostic accuracy than S-ADC in differentiating a tubo-ovarian mass from an ovarian malignancy

ing TOA [20]. It is worth mentioning that the "penumbra sign" is not specific to TOA and can be seen in subacute haemorrhage, which can explain its presence in 2 of the malignant lesions in our study [21].

An abscess cavity contains pus that consists of proteinaceous materials, inflammatory cells, bacteria, and cellular debris. The amino acid groups of these macromolecules are bound to water molecules in a suppurative environment that hinders the motion of the water molecules.
Altogether these factors result in diffusion restriction with low ADC values [22]. This feature is very useful in the diagnosis of brain abscess and liver abscess [23,24]. All the TOAs in our study showed diffusion restriction with low ADC values, reflecting the purulent and viscous contents within the abscess cavity. Conversely, cystic and necrotic areas of tumours usually contain less viscous fluid or debris with only a few inflammatory cells and thus exhibit a greater degree of movement of the water molecules, 
Table 6. Sensitivity and specificity of the "penumbra sign", diffusion restriction, and ADC values in differentiating TOA from ovarian malignancy

\begin{tabular}{|l|c|c|}
\hline Variable & Sensitivity (\%) & Specificity (\%) \\
\hline Penumbra sign & 87.5 & 89.5 \\
\hline \begin{tabular}{l} 
Cystic components \\
\hline Diffusion restriction
\end{tabular} & 100 & 89.5 \\
\hline C-ADC (cut-off: $\left.1.31 \times 10^{-3} \mathrm{~mm}^{2} / \mathrm{s}\right)$ & 95.8 & 89.5 \\
\hline Solid components & 100 & 79.2 \\
\hline Diffusion restriction & 80.8 & 79.2 \\
\hline S-ADC (cut-off: $\left.0.869 \times 10^{-3} \mathrm{~mm}^{2} / \mathrm{s}\right)$ & & \\
\hline
\end{tabular}

causing no or a lesser degree of diffusion restriction with higher ADC values [22]. This feature has been utilized in differentiating a necrotic brain tumour from a brain abscess $[12,23]$. The majority of ovarian malignancies did not show diffusion restriction in their cystic part, except for 1 granulosa cell tumour and 1 serous cystadenocarcinoma in our study. Diffusion restriction in these 2 neoplasms $(10.5 \%)$ was due to haemorrhage, revealed by their marked T1 hyperintensity. Wang et al. reported diffusion restriction in the cystic part of all the included TOAs $(n=34)$, whereas only $11.4 \%$ of ovarian malignancies exhibited diffusion restriction due to haemorrhage and debris [15]. Fan et al. and Tantawy et al. also demonstrated diffusion restriction in the cystic part of all the TOAs $[10,25]$. Another study by Chou et al. revealed diffusion restriction within the cavities of $91 \%$ pelvic abscess $(n=23)$, whereas facilitated diffusion was found in cystic components of pelvic tumours [16]. Interestingly, other cystic benign lesions like endometrioma and ovarian dermoid can also show diffusion restriction due to haemorrhagic and keratinoid materials, respectively [26,27]. Nevertheless, they rarely create diagnostic confusion due to their typical imaging features on MRI.

The current study revealed a significantly lower c-ADC value in TOAs than ovarian malignancies $\left(0.779 \times 10^{-3}\right.$ $\mathrm{mm}^{2} / \mathrm{s}$ vs. $\left.2.368 \times 10^{-3} \mathrm{~mm}^{2} / \mathrm{s} ; p<0.001\right)$. A cut-off $\mathrm{c}-\mathrm{ADC}$ value of $1.31 \times 10^{-3} \mathrm{~mm}^{2} / \mathrm{s}$, derived from the ROC curve, had a sensitivity of $95.8 \%$ and specificity of $89.5 \%$ in differentiating TOA from ovarian malignancy. The mean c-ADC value of TOAs reported in different studies ranges from 0.9 to $1.1 \times 10^{-3} \mathrm{~mm}^{2} / \mathrm{s}$, except for a study by Tantawy et al., who reported a lower c-ADC value of $0.63 \times$ $10^{-3} \mathrm{~mm}^{2} / \mathrm{s}$, whereas cystic areas in ovarian malignancies consistently showed a very high mean C-ADC value of $>2.0 \times 10^{-3} \mathrm{~mm}^{2} / \mathrm{s}$ across the different studies $[10,15,16,25]$. It is worth mentioning that the ADC value within the abscess cavity varies at different stages [28], which could be an important reason behind the variable c-ADC values from study to study. In the early stage, pus has low viscosity and contains fewer inflammatory cells, and thus confers less restriction in the motion of water molecules. A study by Holzapfel et al. illustrated that the mean ADC value in mature abscesses $\left(0.3\right.$ to $\left.0.7 \times 10^{-3} \mathrm{~mm}^{2} / \mathrm{s}\right)$ was lower compared to early abscesses (1.2 to $\left.1.6 \times 10^{-3} \mathrm{~mm}^{2} / \mathrm{s}\right)$ [28]. Additionally, diffusion characteristics are also affected by the chronicity of an abscess and antibiotic therapy. Diffusion restriction may not be seen at all in a chronic and antibiotic-treated abscess [29].

With disease progression, TOAs show enhancing solid tissue, wall or septal thickening, and even papillary projections due to the formation of abundant granulation tissue and fibrous tissue hyperplasia $[4,30]$. It is known that tissue cellularity, interstitial oedema, and the fibrous component can influence the diffusion signal and ADC values [31]. We speculate that the proportion of tissue cellularity, oedema, and fibrous tissue component varies depending on the chronicity of the abscess, which could be the reason for variable diffusion signal and ADC values among TOAs. Only 20.8\% TOAs in our study cohort showed diffusion restriction in the solid part. In contrast, diffusion restriction was seen in the solid components of all ovarian malignancies in our study. The malignant tissues have high cellular density, and cell volumes with a paucity of interstitial oedema results in a higher degree of diffusion restriction and lower $\mathrm{ADC}$ values [11].

The mean s-ADC value of the TOA group was 1.041 $\times 10^{-3} \mathrm{~mm}^{2} / \mathrm{s}$, and that of the ovarian malignancy group was $0.760 \times 10^{-3} \mathrm{~mm}^{2} / \mathrm{s}$. This difference in $\mathrm{s}-\mathrm{ADC}$ value was statistically significant $(p<0.001)$. A cut-off s-ADC value of $0.869 \times 10^{-3} \mathrm{~mm}^{2} / \mathrm{s}$ had a sensitivity of $80.8 \%$ and specificity of $79.2 \%$ in differentiating TOA from ovarian malignancy. The low specificity was due to the overlap of s-ADC values between TOAs and ovarian malignancies. A similar pattern of diffusion behaviour was seen in the study by Wang et al., which demonstrated diffusion restriction in solid components of $46.2 \%(12 / 26)$ TOAs with a mean s-ADC value of $1.43 \times 10^{-3} \mathrm{~mm}^{2} / \mathrm{s}$, and diffusion restriction was shown by all 26 ovarian malignancies with a mean s-ADC value of $1.18 \times 10^{-3} \mathrm{~mm}^{2} / \mathrm{s}$ $(p<0.05)$ [15].

The current study showed a reasonably good diagnostic value of the "penumbra sign", diffusion restriction, and $\mathrm{ADC}$ value of solid and cystic areas in differentiating TOA from ovarian malignancy. ADC values of the cystic components had a higher diagnostic accuracy than the ADC values of solid components. 
Our study had a few limitations. First, the study was retrospective with a limited sample size. Secondly, a complete spectrum of ovarian malignancies and other atypical causes of TOA, such as actinomycosis, were not included, which could have influenced our results. Thirdly, a simplified mono-exponential model of only $3 b$ values $(b=50$, 400 , and $800 \mathrm{~s} / \mathrm{mm}^{2}$ ) was used, which may give an incomplete picture of the true diffusion process, thus limiting the accuracy of ADC calculations. Several recent studies have recommended using bi-exponential models to provide more exact information about the diffusion and perfusion fraction in the tissues according to the intravoxel incoherent motion (IVIM) theory [32]. Large multicentre studies are required to validate our findings further.

\section{Conclusions}

"Penumbra sign" on T1W images and diffusion restriction in the cystic components in a complex adnexal mass favours TOA, whereas diffusion restriction in solid components favours ovarian malignancy. ADC values in the cystic components are more accurate than ADC values in solid components while distinguishing TOA from ovarian malignancies.

\section{Conflicts of interest}

The authors report no conflict of interest.

\section{References}

1. Landers DV, Sweet RL. Tubo-ovarian abscess: contemporary approach to management. Rev Infect Dis $1983 ; 5:$ 876-884.

2. Lareau SM, Beigi RH. Pelvic inflammatory disease and tubo-ovarian abscess. Infect Dis Clin North Am 2008; 22: 693-708.

3. But I, Reljic M. The value of serum CA 125 for the management of tubo-ovarian abscesses. Wien Klin Wochenschr 2000; 112: 1044 1048.

4. Ha HK, Lim GY, Cha ES, et al. MR imaging of tubo-ovarian abscess. Acta Radiol 1995; 36: 510-514.

5. Rakheja R, Makis W, Hickeson M. Bilateral tubo-ovarian abscess mimics ovarian cancer on MRI and (18)F-FDG PET/CT. Nucl Med Mol Imaging 2011; 45: 223-228.

6. Curry A, Williams T, Penny ML. Pelvic inflammatory disease: diagnosis, management, and prevention. Am Fam Physician 2019; 100: 357-364.

7. Garcia-Eulate R, Hussain N, Heller T, et al. CT and MRI of hepatic abscess in patients with chronic granulomatous disease. AJR Am J Roentgenol 2006; 187: 482-490.

8. Grey AC, Davies AM, Mangham DC, Grimer RJ, Ritchie DA. The 'penumbra sign' on T1-weighted MR imaging in subacute osteomyelitis: frequency, cause and significance. Clin Radiol 1998; 53: 587-592.

9. Foti PV, Attinà G, Spadola $S$, et al. MR imaging of ovarian masses: classification and differential diagnosis. Insights Imaging 2016; 7: 21-41.

10. Fan H, Wang TT, Ren G, et al. Characterization of tubo-ovarian abscess mimicking adnexal masses: Comparison between contrastenhanced CT, ${ }^{18} \mathrm{~F}-\mathrm{FDG}$ PET/CT and MRI. Taiwan J Obstet Gynecol 2018; 57: 40-46.

11. Thomassin-Naggara I, Toussaint I, Perrot N, et al. Characterization of complex adnexal masses: value of adding perfusion- and diffusion-weighted MR imaging to conventional MR imaging. Radiology 2011; 258: 793-803.

12. Ebisu T, Tanaka C, Umeda M, et al. Discrimination of brain abscess from necrotic or cystic tumors by diffusion-weighted echo planar imaging. Magn Reson Imaging 1996; 14: 1113-1116.

13. Kim HJ, Lee SY, Shin YR, Park CS, Kim K. The value of diffusion-weighted imaging in the differential diagnosis of ovarian le- sions: a meta-analysis. PLoS One 2016; 11: e0149465. doi: 10.1371/ journal.pone.0149465.

14. Pi S, Cao R, Qiang JW, Guo YH. Utility of DWI with quantitative ADC values in ovarian tumors: a meta-analysis of diagnostic test performance. Acta Radiol 2018; 59: 1386-1394.

15. Wang T, Li W, Wu X, et al. Tubo-ovarian abscess (with/without pseudotumor area) mimicking ovarian malignancy: role of diffusion-weighted MR imaging with apparent diffusion coefficient values. PLoS One 2016; 11: e0149318. doi: 10.1371/journal.pone.0149318.

16. Chou CP, Chiou SH, Levenson RB, et al. Differentiation between pelvic abscesses and pelvic tumors with diffusion-weighted MR imaging: a preliminary study. Clin Imaging 2012; 36: 532-538.

17. Timmerman D, Ameye L, Fischerova D, et al. Simple ultrasound rules to distinguish between benign and malignant adnexal masses before surgery: prospective validation by IOTA group. BMJ 2010; 341: c6839. doi: 10.1136/bmj.c6839.

18. Foti PV, Ognibene N, Spadola S, et al. Non-neoplastic diseases of the fallopian tube: MR imaging with emphasis on diffusion-weighted imaging. Insights Imaging 2016; 7: 311-327.

19. Bilgin T, Karabay A, Dolar E, Develioğlu OH. Peritoneal tuberculosis with pelvic abdominal mass, ascites and elevated CA 125 mimicking advanced ovarian carcinoma: a series of 10 cases. Int J Gynecol Cancer 2001; 11: 290-294.

20. Kim SH, Kim SH, Yang DM, Kim KA. Unusual causes of tuboovarian abscess: CT and MR imaging findings. Radiographics 2004; 24: 1575-1589.

21. Imaoka I, Wada A, Kaji Y, et al. Developing an MR imaging strategy for diagnosis of ovarian masses. Radiographics 2006; 26: 1431-1448.

22. Castillo M, Mukherji SK. Diffusion-weighted imaging in the evaluation of intracranial lesions. Semin Ultrasound CT MR 2000; 21 : 405-416.

23. Chiang IC, Hsieh TJ, Chiu ML, Liu GC, Kuo YT, Lin WC. Distinction between pyogenic brain abscess and necrotic brain tumour using 3-tesla MR spectroscopy, diffusion and perfusion imaging. Br J Radiol 2009; 82: 813-820.

24. Chan JH, Tsui EY, Luk SH, et al. Diffusion-weighted MR imaging of the liver: distinguishing hepatic abscess from cystic or necrotic tumor. Abdom Imaging 2001; 26: 161-165. 
25. Tantawy MSI, Elrakhawy MM, El-Morsy A, et al. DWI in characterization of complex ovarian masses, would it help? The Egyptian Journal of Radiology and Nuclear Medicine 2018; 49: 878-883.

26. Fujii S, Kakite S, Nishihara K, et al. Diagnostic accuracy of diffusionweighted imaging in differentiating benign from malignant ovarian lesions. J Magn Reson Imaging 2008; 28: 1149-1156.

27. Nakayama T, Yoshimitsu K, Irie H, et al. Diffusion-weighted echoplanar MR imaging and ADC mapping in the differential diagnosis of ovarian cystic masses: usefulness of detecting keratinoid substances in mature cystic teratomas. J Magn Reson Imaging 2005; 22: 271-278.

28. Holzapfel K, Rummeny E, Gaa J. Diffusion-weighted MR imaging of hepatic abscesses: possibility of different apparent diffusion coef ficient (ADC)-values in early and mature abscess formation. Abdom Imaging 2007; 32: 538-539.
29. Unal O, Koparan HI, Avcu S, Kalender AM, Kisli E. The diagnostic value of diffusion-weighted magnetic resonance imaging in soft tissue abscesses. Eur J Radiol 2011; 77: 490-494.

30. Ueda H, Togashi K, Kataoka ML, et al. Adnexal masses caused by pelvic inflammatory disease: MR appearance. Magn Reson Med Sci 2002; 1: 207-215

31. Qayyum A. Diffusion-weighted imaging in the abdomen and pelvis: concepts and applications. Radiographics 2009; 29: 1797-1810.

32. Orton MR, Messiou C, Collins D, et al. Diffusion-weighted MR imaging of metastatic abdominal and pelvic tumours is sensitive to early changes induced by a VEGF inhibitor using alternative diffusion attenuation models. Eur Radiol 2016; 26: 1412-1419. 